\title{
Organ of Corti Potentials and the Motion of the Basilar Membrane
}

\author{
Anders Fridberger, ${ }^{1}$ Jacques Boutet de Monvel, ${ }^{1}$ Jiefu Zheng, ${ }^{2}$ Ning Hu, ${ }^{2}$ Yuan Zou, ${ }^{2}$ Tianying Ren, ${ }^{2}$ and Alfred Nuttall ${ }^{2,3}$ \\ ${ }^{1}$ Center for Hearing and Communication Research, Departments of Clinical Neuroscience and Otolaryngology, Karolinska Institutet, SE-171 76 Stockholm, \\ Sweden, ${ }^{2}$ Department of Otolaryngology-Head and Neck Surgery, Oregon Health and Science University, Oregon Hearing Research Center, Portland, \\ Oregon 97201-3098, and ${ }^{3}$ Kresge Hearing Research Institute, The University of Michigan, Ann Arbor, Michigan 48109-0506
}

\begin{abstract}
During sound stimulation, receptor potentials are generated within the sensory hair cells of the cochlea. Prevailing theory states that outer hair cells use the potential-sensitive motor protein prestin to convert receptor potentials into fast alterations of cellular length or stiffness that boost hearing sensitivity almost 1000 -fold. However, receptor potentials are attenuated by the filter formed by the capacitance and resistance of the membrane of the cell. This attenuation would limit cellular motility at high stimulus frequencies, rendering the above scheme ineffective. Therefore, Dallos and Evans (1995a) proposed that extracellular potential changes within the organ of Corti could drive cellular motor proteins. These extracellular potentials are not filtered by the membrane. To test this theory, both electric potentials inside the organ of Corti and basilar membrane vibration were measured in response to acoustic stimulation. Vibrations were measured at sites very close to those interrogated by the recording electrode using laser interferometry. Close comparison of the measured electrical and mechanical tuning curves and time waveforms and their phase relationships revealed that those extracellular potentials indeed could drive outer hair cell motors. However, to achieve the sharp frequency tuning that characterizes the basilar membrane, additional mechanical processing must occur inside the organ of Corti.
\end{abstract}

Key words: cochlea; basilar membrane; laser interferometry; guinea pig; electromotility; outer hair cells

\section{Introduction}

During sound-evoked vibration of the hearing organ, the gating of mechanically sensitive ion channels on the sensory cells produce rapidly alternating electric potentials. One type of sensory cell, the outer hair cell (Fig. 1A), responds to changes in membrane potential with alterations of cellular length and stiffness (Brownell et al., 1985; He and Dallos, 1999). Length and stiffness changes depend on a specialized motor protein, prestin (Zheng et al., 2000). Electrically evoked hair cell motility is fast, with an upper frequency limit of at least $80 \mathrm{kHz}$ (Frank et al., 1999; Grosh et al., 2004). Most theories of hearing organ function assume that force generated by prestin amplifies the motion of the organ, yielding a large increase of auditory sensitivity (Brownell et al., 1985; Ashmore, 1987; Ruggero and Rich, 1991; Evans and Dallos, 1993; Nuttall and Ren, 1995; Liberman et al., 2002; Fridberger and Boutet de Monvel, 2003).

A fundamental problem inherent to this theory is that the resistance and capacitance of the cell membrane of the outer hair cell creates a low-pass filter that attenuates rapid voltage changes.

Received July 7, 2004; revised Sept. 27, 2004; accepted Sept. 27, 2004.

This work was supported by the Swedish Research Council, the Human Frontier Science Program, the Tysta Skolan Foundation, Hörselskadades Riksförbund, the Åke Wiberg Foundation, the Tore Nilson Foundation, the Swedish Society of Medicine, Karolinska Institutet, and National Institutes of Health-National Institute on Deafness and Other Communication Disorders Grants R01 DC04554 and R01 DC00141. We acknowledge Drs. Egbert de Boer and Karl Grosh for helpful discussions.

Correspondence should be addressed to Dr. Alfred L. Nuttall, Oregon Hearing Research Center, 3181 Southwest Sam Jackson Park Road, NRC04, Portland, OR 97201-3098. E-mail: nuttall@ohsu.edu.

DOI:10.1523/JNEUROSCI.2711-04.2004

Copyright $\odot 2004$ Society for Neuroscience $\quad 0270-6474 / 04 / 2410057-07 \$ 15.00 / 0$
Therefore, receptor potentials are too small to induce physiologically relevant motion at stimulus frequencies exceeding a few kilohertz (Fig. 1B,C) (Santos-Sacchi, 1992; Preyer et al., 1996). However, during sound stimulation, fast potential changes occur in the fluid space surrounding the outer hair cells (Geisler et al., 1990; Kössl and Russell, 1992). These voltage changes are generated by the transducer channels, but the exact current path is not fully understood. Their bandwidth much exceeds that of the hair cell receptor potentials. Therefore, Dallos and Evans (1995a,b) proposed that such voltage changes could drive motor proteins, thus alleviating the high-frequency problem.

Their argument hinges on a simple fact: if the fluid surrounding a cell becomes more positive, the cell is in effect hyperpolarized with respect to that same fluid. Consequently, prestin could be driven by the membrane potential changes that may arise as a result of the sound-evoked extracellular potentials mentioned above. Dallos and Evans derived an equivalent circuit for this type of hair cell stimulation, suggesting that little attenuation should occur even at very high stimulus frequencies (Fig. 1D). Some support for the theory came from measurements on isolated hair cells, the motility of which showed a frequency dependence compatible with the proposed equivalent circuit. Experimental evidence from more intact preparations is lacking.

Recently, we measured electric potentials within the organ of Corti and basilar membrane (BM) motion in the same animal (Fridberger et al., 2002b). Such measurements may also be used to shed light on the high-frequency problem by enabling comparison of the input to the system presumed by Dallos and Evans (electric potential) with the output, BM motion. 

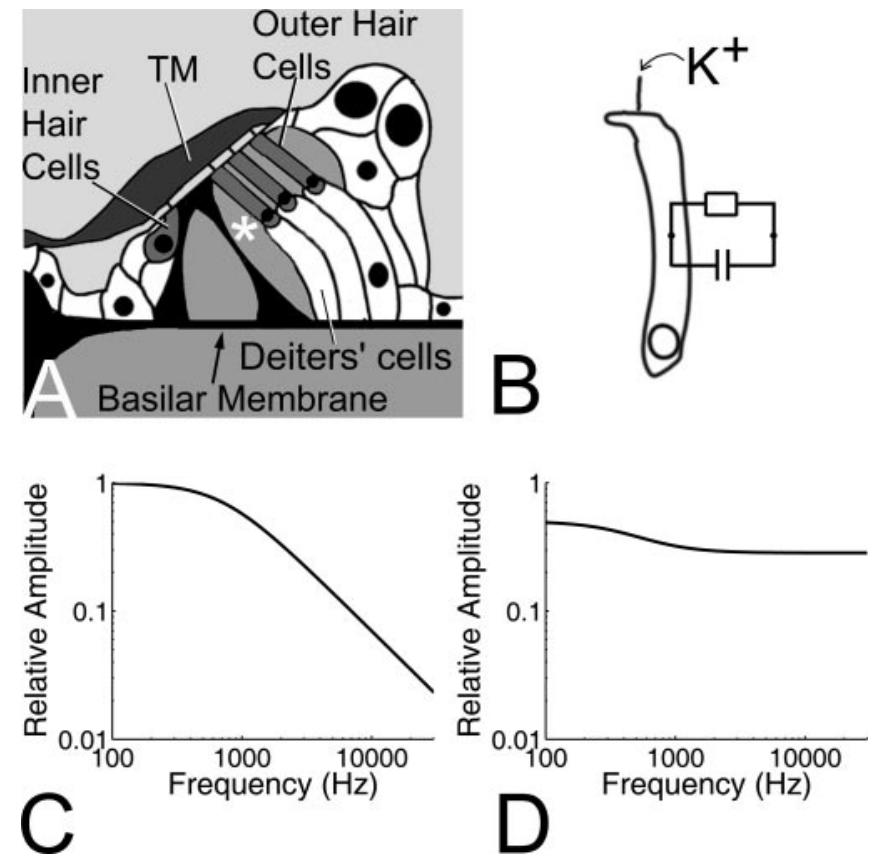

Figure 1. A, Schematic drawing showing major structures in a cross section of the organ of Corti. The white asterisk marks the fluid space where fast potential changes occur. TM, Tectorial membrane. $B$, Receptor current entering through transducer channels will be filtered by the parallel resistance and capacitance of the cell membrane of the outer hair cell. This filter has a frequency response similar to the one shown in C, causing substantial high-frequency attenuation. If extracellular potentials are assumed to drive prestin, the equivalent circuit is more complex, with the frequency response shown in D. In this case, the filter is almost flat throughout the entire frequency range [for parameters and additional details, see Dallos and Evans (1995a)].

\section{Materials and Methods}

Young guinea pigs were anesthetized with ketamine $(40 \mathrm{mg} / \mathrm{kg})$ and xylazine $(10 \mathrm{mg} / \mathrm{kg})$ and prepared for recording of BM velocities, as described previously (Nuttall et al., 1991). All animals were tracheotomized, but artificial ventilation was not used. Animal temperature was continuously monitored with a rectal probe and maintained at $38^{\circ} \mathrm{C}$ using a heating blanket. After exposing the auditory bulla, the compound action potential of the auditory nerve was recorded using a silver ball electrode positioned in the round window niche. Stimuli were gated tone bursts with $10 \mathrm{msec}$ duration and a rise/fall time of $1 \mathrm{msec}$. Only guinea pigs with a normal initial compound action potential audiogram were used in this study. The electrode on the round window was also used to monitor the amplitude of the simple difference tone evoked by continuous low-level stimulation at 17 and $17.9 \mathrm{kHz}$ (i.e., the round window signal at $900 \mathrm{~Hz}$ ). This distortion product is highly sensitive to pathology induced by surgical trauma. Whenever it changed, surgery was temporarily halted to allow recovery.

After creating a wide opening in the bulla, the middle ear muscles were transected, and a small opening was created in the basal cochlear turn. This region of the cochlea had best frequencies in the range of $15-19 \mathrm{kHz}$ when stimulated at low and moderate intensities.

Recording of BM velocity. After opening the basal turn, gold-coated glass beads were placed on the BM to provide a high-reflectance target for the laser velocimeter (OFV1102; Polytec Inc., Waldbronn, Germany). Positive voltage from the velocimeter corresponded to velocity directed at scala tympani.

The frequency response of the velocimeter was measured by focusing the laser on a piezoelectric crystal with a resonance frequency of $200 \mathrm{kHz}$. The velocimeter showed a time delay of $\sim 6 \mu \mathrm{sec}$. All recordings were compensated for this effect.

Sound stimulation. A digital lock-in amplifier (SR830; Stanford Instruments, Sunnyvale, CA) was used to generate stimuli and measure the response. Time domain data were acquired using 16 bit digital-to-analog

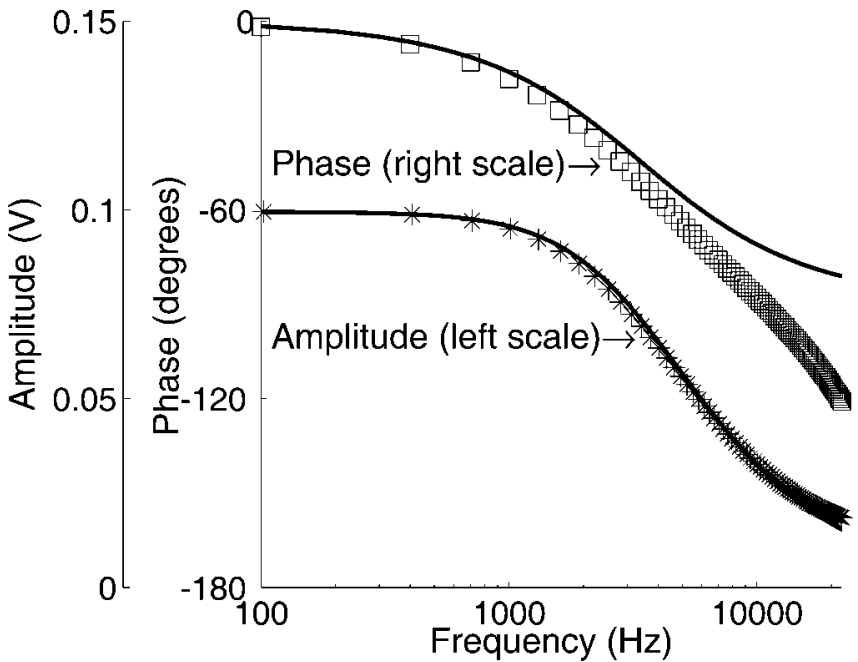

Figure 2. Calibration of the recording system. Asterisks and squares denote, respectively, the measured amplitude and phase of the recording electrode and amplifier. Solid lines are fits of the amplitude data to a first-order filter that in this case had a cutoff frequency of $3.4 \mathrm{kHz}$. The input level was $10 \mathrm{mV}$; amplifier gain of 10 .

and analog-to-digital converters (system II; Tucker-Davies Technologies, Alachua, FL). Stimuli were delivered to the ear through a pair of 0.5 inch condenser microphones driven in reverse by custom power amplifiers. Microphones were housed in an isolated and grounded speculum fitted tightly to the animal's ear canal. Stimulus frequencies ranged from 0.2 to $22 \mathrm{kHz}$ with $100-200 \mathrm{~Hz}$ frequency steps and a lock-in time constant of $30 \mathrm{msec}-1 \mathrm{sec}$, depending on the signal level. Supplemental time domain data used $20 \mathrm{msec}$ tone bursts with a $1 \mathrm{msec}$ rise/fall time, repeated up to 50 times to allow averaging.

Measurement of local electric potentials within the organ of Corti. After quantification of BM motion, a sharp microelectrode was advanced toward the organ of Corti through the opening used for recording mechanical responses. Electrodes were pulled from 1-mm-diameter borosilicate glass capillaries. Tip diameters were slightly $<1 \mu \mathrm{m}$. Because we had no desire to perform intracellular recordings, these relatively large electrodes were used to minimize filtering of electric potentials by the electrode. As electrodes advanced through the scala tympani fluid, voltage changes induced by continuous sound stimulation at $17 \mathrm{kHz}$ were monitored. The amplitude of these potentials increased on approaching the hearing organ. BM penetration was evident through a transient negative voltage followed by an increase of the measured potential evoked by the $17 \mathrm{kHz}$ tone. Potentials were recorded using a BMA-200 amplifier (CWA Inc., Ardmore, PA) connected to the SR830 lock-in amplifier.

As a result of its resistance and capacitance, the electrode filtered the electric potentials. Each electrode was therefore calibrated while still in position within the organ of Corti, using the circuit described by BadenKristensen and Weiss (1983). Figure 2 shows a representative calibration of the recording system (including the amplifier), with settings identical to those used during recording of the organ of Corti potential. Leastsquares fits of the calibration data showed that the system behaved as a single-pole low-pass filter, with $3 \mathrm{~dB}$ cutoff frequencies ranging from 1.6 to $8 \mathrm{kHz}$ (capacitance compensation was not used). Although the amplitude response was that of a first-order filter, the phase deviated from this behavior. The main reason for this discrepancy was that the amplifier introduced additional frequency-dependent phase lag. Filtering properties systematically depended on the recording location. Higher cutoff frequencies were found with the electrode positioned close to the surface of the scala tympani fluid. Cutoff frequencies were lower when positioned inside the organ of Corti. Such effects of fluid depth are well known in electrophysiological recordings, and they necessitate in situ electrode calibration.

After electrode calibration, BM velocities were again measured, using identical stimulus parameters. Electrode penetration frequently induced some loss of auditory sensitivity. Subsequent graphs therefore use BM 


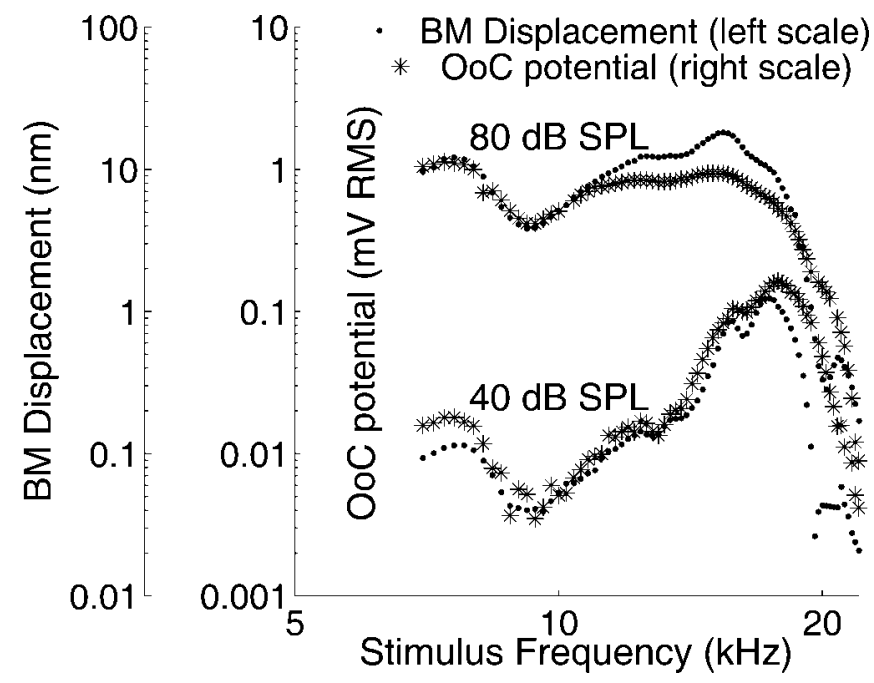

Figure 3. BM displacement (left $y$-scale) and organ of Corti electric potentials ( 00 C; right $y$-scale) as a function of stimulus frequency for two different stimulus levels. Amplitudes were corrected for the response properties of the recording system. Recordings were from a relatively sensitive animal (total sensitivity loss of $10 \mathrm{~dB}$ after withdrawal of recording electrode).

data acquired after withdrawal of the recording electrode, except where otherwise noted.

Signal processing. Data analysis was performed using Matlab (release 14; The MathWorks Inc., Natick, MA). Electrical data were corrected for the frequency response of the electrode and recording system. Time domain data were used to verify the phase relationships found with the lock-in amplifier, because these data allowed the absolute time relationships between electrical potentials and BM motion to be seen, whereas the lock-in amplifier provided a phase value that was always between 180 and $-180^{\circ}$. $Q_{10 \mathrm{db}}$ values were calculated by dividing the stimulus frequency giving the maximal response with the bandwidth $10 \mathrm{~dB}$ below the peak.

Potentials in the organ of Corti fluid spaces are dominated by contributions from outer hair cells, which most likely respond to displacement of the BM (von Békésy, 1951). Therefore, tuning curve amplitudes were converted to displacement by dividing velocity by $2 \pi f$, where $f$ is the stimulus frequency. Because displacement lags velocity by $90^{\circ}$, conversion of phase difference curves is straightforward. In the time domain, velocity can be converted to displacement by cumulative integration, but this process was found to introduce artifacts. Therefore, the time waveforms in Figures 4 and 5 use the measured quantity velocity rather than the computed displacement.

\section{Results}

Tuning curve shape and amplitude

When the electrode tip was positioned at the surface of the fluid in scala tympani, tuning was essentially absent, and amplitudes of the electric potentials were low. Most likely, this occurred because neighboring hair cells may vibrate with very different phase, their microphonic potentials canceling each other. Closer to the BM, the measured potentials should become dominated by a smaller number of generators with similar tuning and phase, resulting in increased amplitude. Curves acquired close to the BM indeed resembled mechanical tuning curves, although the electric potentials were less sharply tuned. The same was true for potentials inside the organ of Corti. Figure 3 shows BM displacement (dots; left $y$-scale) plotted together with the potential inside the organ of Corti (asterisks; right $y$-scale) for two different stimulus levels [40 and $80 \mathrm{~dB}$ sound pressure level (SPL)]. At each level, the curves practically superimpose for low stimulus frequencies. Differences between the curves are found around the peak, especially on the high-frequency side, where the BM curve had a much steeper

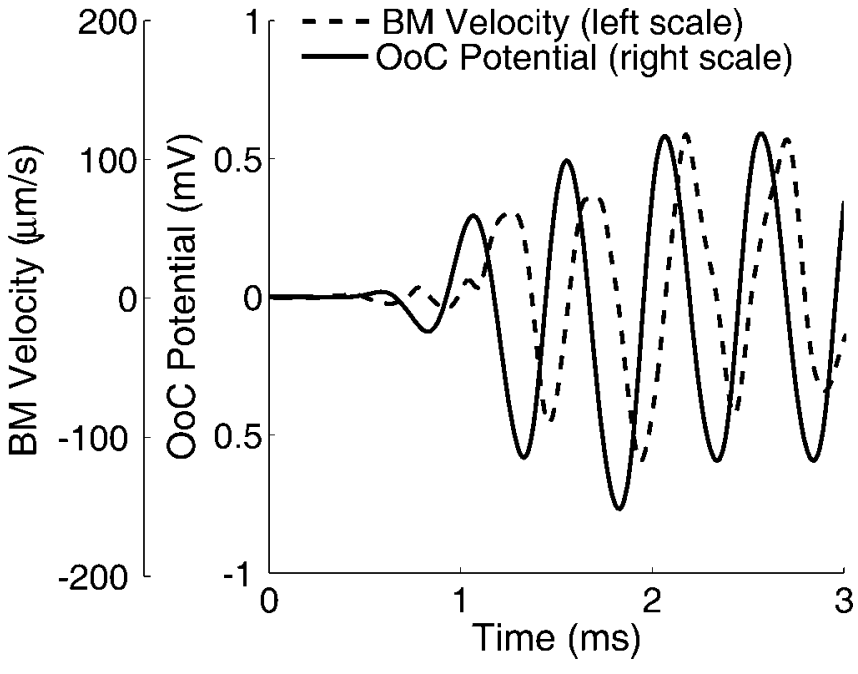

Figure 4. The first 3 msec of the response to a $100 \mathrm{~dB}$ SPL tone burst at $2 \mathrm{kHz}$ (stimulus rise time, $1 \mathrm{msec}$; $\cos ^{2}$ envelope). BM velocity (leftmost $y$-scale; dashed line) is plotted together with organ of Corti potentials ( 00 C; right $y$-scale; solid line). Records were compensated for a 29 $\mu$ sec delay introduced by the recording electrode and a $6 \mu$ sec delay introduced by the velocimeter. Both traces were low-pass filtered offline with a zero-phase fourth-order filter with a $10 \mathrm{kHz}$ cutoff frequency. To prevent ringing artifacts introduced by filtering, amplitudes were reduced to zero at the beginning of the record using a Blackman window. In this sensitive preparation, technical problems prevented recording of the BM velocity after electrode penetration.

slope. This resulted in sharper tuning for the mechanical responses, which at $40 \mathrm{~dB}$ SPL had a $Q_{10 \mathrm{db}}$ value of 4.6 compared with a $Q_{10 \mathrm{db}}$ of 3.6 for the electric potential. Although potentials were recorded at the same location as the bead used for tracing $\mathrm{BM}$ motion, there was a slight difference in the best frequency. At $40 \mathrm{~dB}$ SPL, the electrical tuning curve had its peak response at $17.8 \mathrm{kHz}$ and the BM curve at $17.4 \mathrm{kHz}$, a difference that was more pronounced at lower stimulus levels. Note also the wide bandwidth of the electric potential. There is no sign of a frequency-dependent decrease in amplitude such as that found in outer hair cell receptor potentials (Kössl and Russell, 1992).

Tuning curve shape strongly depended on stimulus level. For the preparation in Figure 3, the organ of Corti potential showed blunt tuning at $80 \mathrm{~dB}$ SPL, and the low-frequency peak at $8 \mathrm{kHz}$ dominated the frequency response. The $40 \mathrm{~dB}$ change of stimulus level resulted in only a $10 \mathrm{~dB}$ increase in amplitude near the peak. These level-dependent variations were smaller for mechanical responses. Although the best frequency moved to a lower frequency, the $8 \mathrm{kHz}$ peak did not become dominant, and the response amplitude increased by $18 \mathrm{~dB}$. Similar data were acquired from one additional sensitive ear. In five less sensitive preparations, tuning curves were flatter and response amplitudes lower. However, even in those preparations, electrical responses were less sharply tuned than their mechanical siblings.

\section{Phase relationships}

If extracellular potentials drive outer hair cell motility, as suggested by Dallos and Evans, it is of interest to examine the temporal relationship between potentials and the motion of the BM (i.e., their relative phase). Figure 4 shows time-domain records acquired with a $100 \mathrm{~dB}$ SPL stimulus at $2 \mathrm{kHz}$. In this and all subsequent figures, positive BM data mean motion directed at scala tympani. Inspection of this graph reveals that electric potentials inside Corti's organ preceded BM velocity. Fourier trans- 


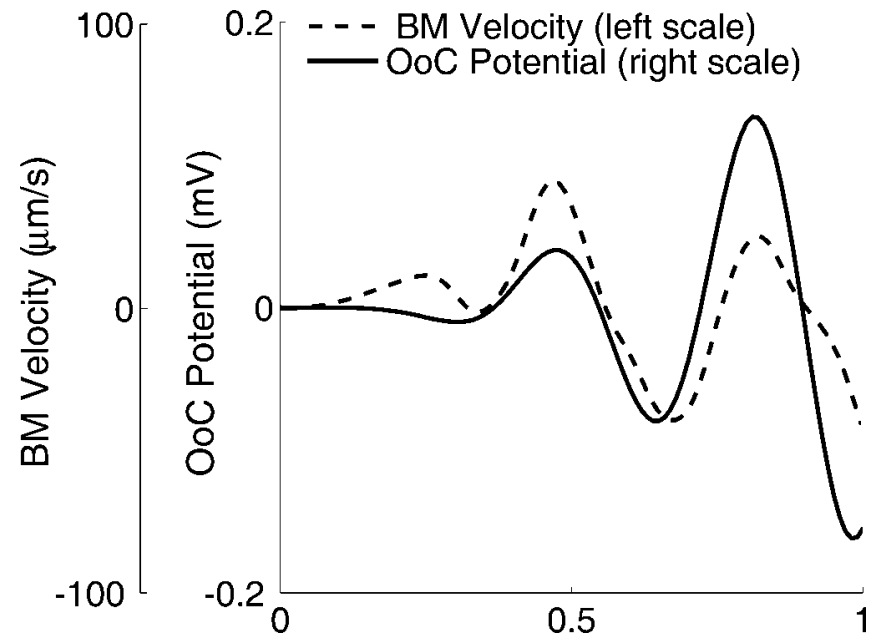

Figure 5. Response onset after a $100 \mathrm{~dB} \mathrm{SPL}$ tone burst at $3 \mathrm{kHz}$. Signals were processed as in Figure 4. $00 \mathrm{C}$, Organ of Corti.

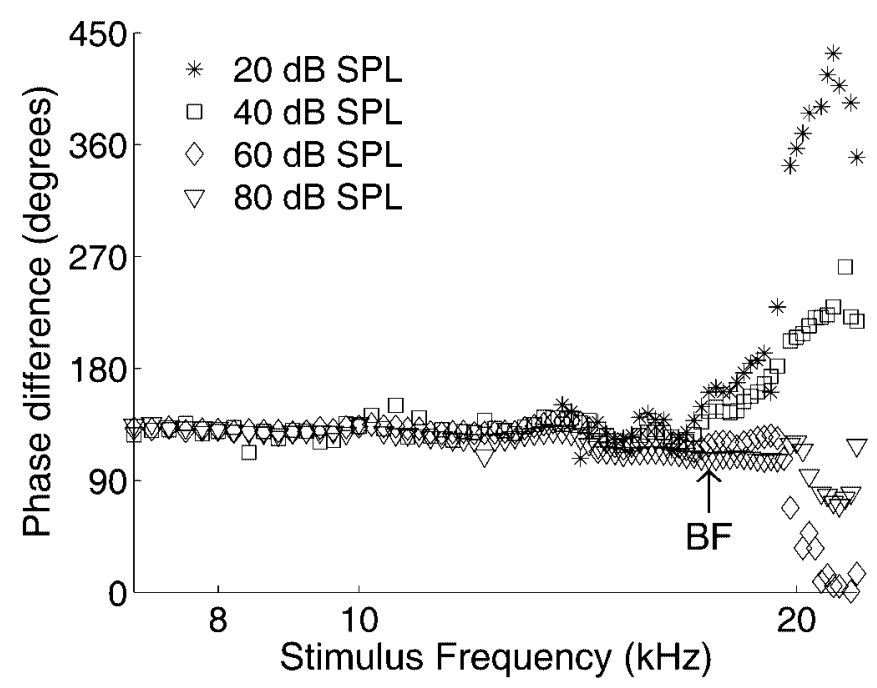

Figure 6. Phase difference between potentials inside the organ of Corti and the BM displacement. Same preparation as in Figure 3. All data were corrected for electrode and velocimeter phase response. BF, Best frequency (the stimulus frequency evoking maximum BM response).

formation gave a phase lead of $59^{\circ}$ after correction for the phase response of the electrode and the velocimeter.

If such an obvious phase lead were present also at the start of the stimulus, it would mean that electric potentials were produced before BM motion. Figure 5 shows that this is not the case. Using a $3 \mathrm{kHz}$ stimulus at $100 \mathrm{~dB}$ SPL, it can be seen that the BM began moving before detectable potentials were generated. Phase modulation occurred thereafter, resulting in a phase lead for the electric potential of $58^{\circ}$.

Figure 6 plots the steady-state phase difference for four different stimulus levels at frequencies between 7 and $22 \mathrm{kHz}$. In this and subsequent figures, computations are based on BM displacement, which lags velocity by $90^{\circ}$; positive phase values mean that electric potentials lead the BM displacement. Note that data in the low-frequency region show little variability (average phase difference for all stimulus levels was $130^{\circ} ; \mathrm{SD}, 5^{\circ}$ ). Well below the best frequency, level dependent phase shifts are absent (Sellick et al., 1982). Because the electrical data underlying this graph were acquired over a 25 min period, this implies that the electrical properties of the animal-electrode interface remained constant

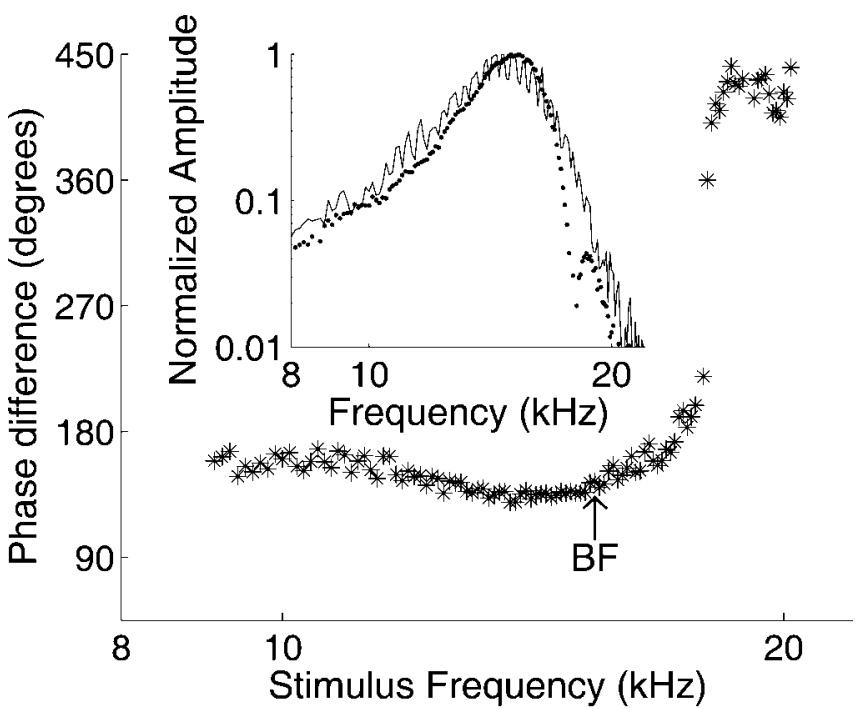

Figure 7. Phase difference between potentials recorded close to the BM and the BM displacement in an animal with a $5 \mathrm{~dB}$ loss of sensitivity at the time of the recording. Stimulus level is $60 \mathrm{dBSPL}$. The inset shows the normalized amplitude of the electric potentials (solid line) and the BM motion (dotted line). $Q_{10 \mathrm{~dB}}$ values of 2.6 for electric potential and 3.7 for the BM. BF, Best frequency.

through this time period. Phase difference curves began diverging at $\sim 15 \mathrm{kHz}$; the peak response amplitude was found at $17.4 \mathrm{kHz}$. At frequencies higher than this, data acquired at 20 and $40 \mathrm{~dB}$ SPL had a larger phase lead than those at 60 and $80 \mathrm{~dB}$ SPL. The $20 \mathrm{~dB}$ SPL curve reached a maximum phase lead in excess of a full cycle, whereas the $80 \mathrm{~dB}$ SPL curve lagged the low-frequency responses.

Data were also acquired from animals with substantially larger loss of auditory sensitivity. The basic pattern of phase difference also persisted in these preparations. A nearly constant phase difference was found well below the best frequency; closer to the peak of the tuning curve, the phase difference increased. Leveldependent phase changes of the kind shown in Figure 6 were only found in the best preparations. In the seven preparations that contributed substantial tuning curve data, the average phase difference in the $10-12 \mathrm{kHz}$ region was $141^{\circ}\left(\mathrm{SD}, 27^{\circ}\right.$; much of this variation came from poor preparations, which had worse signalto-noise ratios).

It is possible that these phase relationships were influenced by the presence of the electrode that penetrated the BM. Therefore, electrical tuning curves were recorded at locations very close to the $\mathrm{BM}$, before actual penetration (three preparations, two of which had $<10 \mathrm{~dB}$ sensitivity loss at the time of the recording). In these recordings, the cochlea is in a close to undisturbed state. The inset in Figure 7 shows one such electrical tuning curve plotted together with its mechanical counterpart (both curves were normalized to a peak value of 1). Again, electrical tuning curves were less sharply tuned than the mechanical ones. Phase difference curves had characteristics similar to those found when the electrode was positioned inside the organ (Fig. 7, main graph). At frequencies $\sim 10 \mathrm{kHz}$, the average phase difference was $158^{\circ}$. A slightly decreased phase lead was seen on approaching the best frequency $(15.4 \mathrm{kHz})$. At frequencies higher than this, electric potentials showed an increasing phase lead over the BM displacement.

\section{Theoretical results}

Phase differences close to and above the best frequency might arise by interference between potentials generated at sites adja- 
cent to each other along the BM. To test this, a mathematical model was designed, using the assumption that the transduction current at a particular cochlear location was proportional to BM displacement. To estimate how the resulting potentials spread within the fluid spaces inside the organ of Corti, this compartment was regarded as a long cylinder with homogeneous electrical properties, enclosing a conducting fluid core provided by perilymph. Cable theory (Hodgkin and Rushton, 1946) then predicts that a sinusoidal source of current of complex amplitude $I_{0}$ and frequency $f$ generates a sinusoidal potential whose amplitude $V(x)$ at distance $x$ from the source is affected by the following complex attenuation factor:

$$
V(x) \propto \exp (-x / \kappa) I_{0} .
$$

Here, the complex number $\kappa$ characterizes the electrical properties per unit length (tissue resistance, capacitance, and the conductivity of fluids inside the organ of Corti). In terms of the space constant $\lambda$ and the time constant $\tau$ of the cable, $\kappa$ is given by the following:

$$
\kappa=\lambda /\left(1+\omega^{2} \tau^{2}\right)^{1 / 4} \exp (-\mathrm{i} \theta / 2), \theta=\arctan (\omega \tau), \omega=2 \pi f .
$$

In other words, $\kappa$ is the square root of $\lambda^{2} /(1+i \omega \tau)$ having a positive real part. The potential recorded by the electrode at a location $x_{0}$ and a particular frequency $\omega$ is modeled by summing the contributions from different points $x$ along the cochlear partition, as follows:

$$
\begin{array}{r}
V_{\text {model }}\left(x_{0}, \omega\right) \propto \int \exp \left(-\left|x-x_{0}\right| / \kappa\right) I_{0}(x, \omega) d x \propto-i / \omega \\
\int \exp \left(-\left|x-x_{0}\right| / \kappa\right) v_{\mathrm{BM}}(x, \omega) d x,
\end{array}
$$

where $v_{\mathrm{BM}}(x, \omega)$ denotes the complex amplitude of the velocity of the BM (counted positive toward scala vestibuli) at point $x$ and frequency $\omega$. We have used the relationship $I_{0}(x, \omega) \propto-i / \omega$ $v_{\mathrm{BM}}(x, \omega)$, which follows from the above assumptions.

To test the above model and obtain an estimate of the constant $\kappa$, we need to know the BM velocity profile for a range of locations adjacent to the measurement site $x_{0}$. The only such data presently available to us are the measurements from the gerbil by Ren (2002). Using these measurements, the right-hand side of Equation 3 allowed us to compute a model potential amplitude $V_{\text {model }}$ gerbil $(y, \omega)$ for locations $y$ along the first turn of the gerbil cochlea as a function of frequency and for any given value of the constant $\kappa$. To compare this model with potential measurements in the guinea pig, we assumed that a simple linear rescaling in frequency could be used to match the gerbil data to the guinea pig data. We then obtained an estimate of $\kappa$ by performing a least-squares fit of the rescaled model impedance $z_{\text {model }}(\omega)=V_{\text {model }}{ }^{\text {gerbil }}\left(y_{0}, a \omega+\right.$ $b) /\left(-i v_{\mathrm{BM}}{ }^{\text {gerbil }}\left(y_{0}, a \omega+b\right)\right)$ to the measured impedance, $z_{\text {meas }}(\omega)=V_{\text {meas }}{ }^{\mathrm{gp}}\left(x_{0}, \omega\right) /\left(-i v_{\mathrm{BM}}{ }^{\mathrm{gp}}\left(x_{0}, \omega\right)\right)$, for the set of frequencies used in the experiments, the constants $a$ and $b$ denoting the linear rescaling used to fit the gerbil data to the guinea pig tuning curve.

The results of the above fitting procedure are illustrated in Figure 8 . The measurement location $x_{0}$ in the guinea pig cochlea had a best frequency of $15.4 \mathrm{kHz}$. The location $y_{0}$ in the gerbil cochlea that best matched $x_{0}$ was situated at a distance of $2.53 \mathrm{~mm}$ from the base with a best frequency of $14 \mathrm{kHz}$. By fitting the

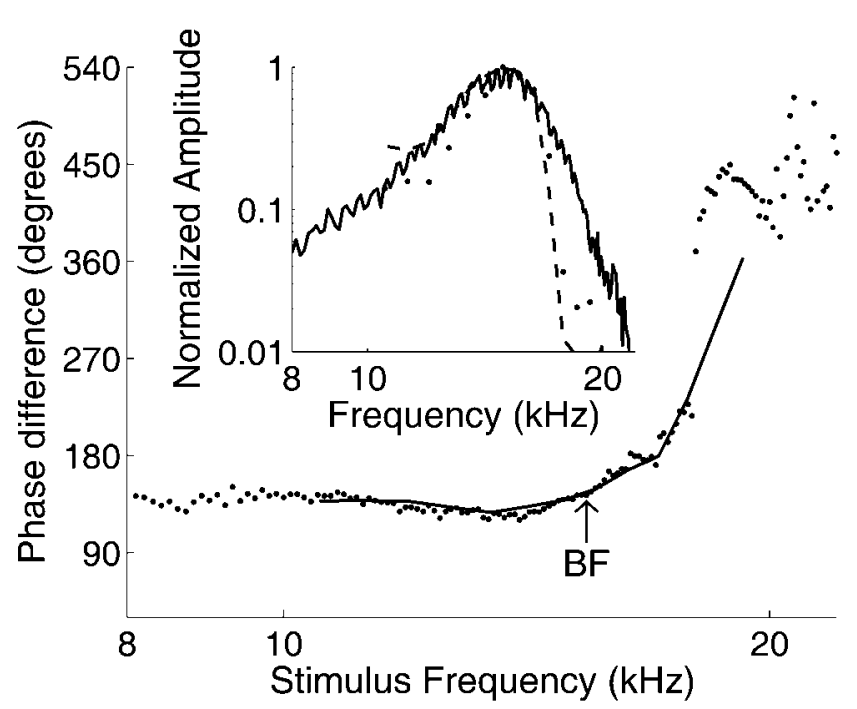

Figure 8. Phase differences between the organ of Corti potential and the BM displacement were predicted by the model described in the text. The dots show the measured phase difference; the solid line is the fitted curve. The inset shows the tuning curves for the BM (dots), the electric potential (solid line), and the fitted curve (dashed line). Note that the model accurately predicted the shape of the electric tuning curve in the frequency region below and slightly above the best frequency. At frequencies higher than this, substantial deviations were found. To verify these results, we also performed curve fits using guinea pig tuning curves mapped to specific cochlear locations using Greenwood's place-frequency map (Greenwood, 1990). Space constants obtained by this modified model were similar to those shown here.

tuning curves over frequencies below $22 \mathrm{kHz}$, we obtained a value of $\kappa$ corresponding to an effective space constant of $40 \mu \mathrm{m}$. Values for the time constant $\tau$ were quite small $(\sim 1 \mu \mathrm{sec})$; this parameter actually had little effect on the fit. Importantly, the model predicted a rapidly increasing phase difference in the region around the best frequency. The model also predicted the general shape of the tuning curves (Fig. 8, inset). Similar fitting results were obtained for the $20 \mathrm{~dB}$ SPL data shown in Figure 6 and the data from Figure 7; in both cases, space constants were in the same range as in Figure 8. Thus, this simplified analysis suggests that the phase differences that developed around the best frequency were caused by interference effects between several distributed generators, each having a rapidly varying phase. Such behavior is expected from the BM at these frequencies.

To verify a fundamental aspect of this model, the BM was penetrated with a current injection electrode and a recording electrode. Electrode separation was systematically altered by moving the recording electrode closer to the current injection site. Square current pulses with $500 \mathrm{msec}$ duration were injected and the resulting potential measured at the end of the current step. Least-squares fits were performed using the relationship $V=k \exp (-x / \lambda)$, where $V$ is the measured potential, $x$ is the electrode separation, and $\lambda$ is the space constant. This experiment showed that potentials declined exponentially with increasing distance and gave a value for $\lambda$ of $42 \mu \mathrm{m}$, consistent both with the model results detailed above and previous estimates $(<300 \mu \mathrm{m})$ (Geisler et al., 1990).

\section{Discussion}

The Dallos-Evans theory

The kernel of this theory is that potential changes in the fluid space surrounding outer hair cells would drive cellular motor proteins. The mathematical analysis presented by Dallos and Evans suggests that such potentials could have a bandwidth much 
larger than that of the hair cell receptor potentials, thereby alleviating the high-frequency problem described in the Introduction (Fig. 1). In our experiments, close examination of the onset response to tone bursts revealed that the BM began moving before detectable electric potentials were produced. Phase modulation occurred thereafter, resulting in a final phase lead between 130 and $160^{\circ}$ for the electric potential. Such behavior is expected if electric potential changes in the fluid space surrounding outer hair cells drive cellular electromotility.

Our most sensitive preparation, with a $10 \mathrm{~dB}$ loss of auditory sensitivity, had maximum amplitude of $27 \mu \mathrm{V}$ at $17 \mathrm{kHz}$ at $20 \mathrm{~dB}$ SPL after correction for attenuation caused by the recording electrode. At such low stimulus intensities, the response of the organ is linear. Taking account of the $10 \mathrm{~dB}$ loss, this implies that the amplitude of the electric potentials would be $\sim 8 \mu \mathrm{V}$ for $0 \mathrm{~dB}$ SPL stimuli at the best frequency. These amplitudes are similar to the ones reported by Kössl and Russell (1992). Measurements from isolated outer hair cells show motility "gain" in the range of 2-30 $\mathrm{nm}$ per $\mathrm{mV}$ (Santos-Sacchi and Dilger, 1988; Santos-Sacchi, 1989). That is, for each millivolt of membrane potential change, isolated cells change their length by $2-30 \mathrm{~nm}$ on average. Because outer hair cells may be affected by the isolation procedure, a relatively conservative value of $20 \mathrm{~nm}$ per $\mathrm{mV}$ may be adopted. Furthermore, assuming that the effective stimulus is $20 \%$ of the measured extracellular response (Dallos and Evans, 1995a), we arrive at an outer hair cell motility of $\sim 0.03 \mathrm{~nm}$ at $0 \mathrm{~dB}$ SPL. This is in the same range as the $\mathrm{BM}$ displacement measured in sensitive cochleae $(\sim 0.02 \mathrm{~nm})$ (Cooper, 1998). Therefore, the potentials that we measured could be large enough to drive outer hair cell motility. In a system with positive feedback, a small amount of feedback may suffice to generate a relatively large response. Therefore, outer hair cells conceivably could provide effective enhancement of BM vibration at even lower amplitudes of the driving stimulus than those assumed here.

Our data imply that certain additional elements would have to be included in the theory. The correspondence between electrical and mechanical tuning curves at low frequencies attest to their close relationship, but the relatively poor tuning of electric potentials near the best frequency means that additional frequencyselective elements are necessary to generate the tuning curves measured from the BM. Such sharpening could be a property inherent to the "cochlear amplifier" itself or be caused by the passive mechanical properties of surrounding structures (supporting cells or the BM itself).

Below the best frequency, the phase difference shown in Figures 6 and 7 was essentially independent of the stimulus frequency. This result has a parallel in recent cochlear models (de Boer and Nuttall, 2003), which find that realistic BM tuning curves can be achieved by mechanical feedforward and feedbackward of energy lacking frequency-dependent phase shift. If we assume that the Dallos-Evans theory is correct, such mechanical effects could be produced by hair cells driven by the extracellular potentials that we characterize here.

The rapid increase of phase difference between the BM and the electric potentials at and above the best frequency is also important. Although this pattern of phase change may be caused by interactions between different adjacent generators (Fig. 8), these potentials would still be the effective stimulus driving outer hair cell motility. Therefore, an increasing phase lead for the electric potentials would have to be compensated by an equally large phase change of outer hair cell motility to generate the BM tuning curves that we measured. Such frequency-dependent phase change was not found in studies of outer hair cell motility in vitro
(Frank et al., 1999). Conceivably, passive mechanical elements within the organ could introduce such a phase lag. To explain, outer hair cells connect to the BM through the cups of the Deiters' cells. Thus, hair cell motility is coupled to the BM through a cellular structure that must possess stiffness, mass, and damping. This would result in a mechanical filter that may affect both amplitude and phase in a frequency-dependent manner.

Extracellular potentials within the organ of Corti represent the averaged response of a group of hair cells. This spatial averaging could be an important property. van Netten et al. (2003) found that the minimum hair bundle deflection that could be reliably detected in the receptor current of the cell was $\sim 5 \mathrm{~nm}$. This number, approximately two orders of magnitude larger than the BM displacement at threshold, is determined primarily by noise inherent to the transduction apparatus. Obviously, spatial averaging could render the system less susceptible to such noise and improve the detection limit, as suggested by van Netten et al. (2003).

\section{Micromechanics of the organ of Corti}

There is near-universal agreement that electric potentials within Corti's organ are generated by stereocilia displacement (Flock, 1965; Hudspeth and Corey, 1977). In vitro measurements from hemicochlea preparations show that opening of mechanically sensitive ion channels occur when the organ of Corti is displaced toward scala vestibuli (at least at low stimulus frequencies) (von Békésy, 1951; He et al., 2004). In our experiments, electrical and mechanical amplitude tuning curves practically superimposed below $10 \mathrm{kHz}$, suggesting that electric potentials were related to BM displacement. Furthermore, the observed phase relationship between BM displacement and electric potential means that potentials are almost, but not exactly, in phase with BM displacement toward scala vestibuli. This discrepancy could be explained by several factors. Because we do not know the exact current path that generates the organ of Corti potential, it is possible that some element of the circuit causes the phase difference. Complex interactions between stereocilia and the tectorial membrane or fast structural alterations within the organ may also be important (Zwislocki and Kletsky, 1979; cf. Ulfendahl et al., 1995; Gummer et al., 1996; Fridberger et al., 2002a; Fridberger and Boutet de Monvel, 2003; Cai et al., 2004). Because of the lateral interference shown in Figure 8, comparisons between the BM and electric potential phases should be performed with caution. At low stimulus frequencies, large sections of the BM vibrate in phase, making this problem less severe.

In summary, confronting the Dallos-Evans model with physiological data shows that extracellular potentials could indeed drive basilar membrane motion. However, substantial sharpening of tuning curves would have to be generated by additional mechanical elements, the exact identity of which remains undefined. In addition, these elements would have to introduce a relatively large phase lag that develops at frequencies around the peak of the tuning curve. These data also imply that complex mechanical interactions may occur between different parts of the organ.

\section{References}

Ashmore JF (1987) A fast motile response in guinea-pig outer hair cells: the cellular basis of the cochlear amplifier. J Physiol (Lond) 388:323-347.

Baden-Kristensen K, Weiss TF (1983) Receptor potentials of lizard hair cells with free-standing stereocilia: responses to acoustic clicks. J Physiol (Lond) 335:699-721

Brownell WE, Bader CR, Bertrand D, de Ribaupierre Y (1985) Evoked me- 
chanical responses of isolated cochlear outer hair cells. Science 227:194-196.

Cai H, Shoelson B, Chadwick RS (2004) Evidence of tectorial membrane radial motion in a propagating mode of a complex cochlear model. Proc Natl Acad Sci USA 101:6243-6248.

Cooper NP (1998) Harmonic distortion on the basilar membrane in the basal turn of the guinea-pig cochlea. J Physiol (Lond) 509:277-288.

Dallos P, Evans BN (1995a) High-frequency motility of outer hair cells and the cochlear amplifier. Science 267:2006-2009.

Dallos P, Evans BN (1995b) High-frequency outer hair cell motility: corrections and addendum. Science 268:1420-1421.

de Boer E, Nuttall AL (2003) Properties of amplifying elements in the cochlea. In: Biophysics of the cochlea: from molecules to models (Gummer AW, Dalhoff E, Nowotny M, Scherer MP, eds), pp 331-342. Singapore: World Scientific.

Evans BN, Dallos P (1993) Stereocilia displacement induced somatic motility of cochlear outer hair cells. Proc Natl Acad Sci USA 90:8347-8351.

Flock $\AA$ (1965) Transducing mechanisms in the lateral line canal organ receptors. Cold Spring Harb Symp Quant Biol 30:133-145.

Frank G, Hemmert W, Gummer AW (1999) Limiting dynamics of highfrequency electromechanical transduction of outer hair cells. Proc Natl Acad Sci USA 96:4420-4425.

Fridberger A, Boutet de Monvel J (2003) Sound-induced differential motion within the hearing organ. Nat Neurosci 6:446-448.

Fridberger A, Boutet de Monvel J, Ulfendahl M (2002a) Internal shearing within the hearing organ evoked by basilar membrane motion. J Neurosci 22:9850-9857.

Fridberger A, Zheng J, Parthasarathi A, Ren T, Nuttall AL (2002b) Loud sound-induced changes in cochlear mechanics. J Neurophysiol 88:2341-2348.

Geisler CD, Yates GK, Patuzzi RB, Johnstone BM (1990) Saturation of outer hair cell receptor currents causes two-tone suppression. Hear Res 44:241-256.

Greenwood DD (1990) A cochlear frequency-position function for several species-29 years later. J Acoust Soc Am 87:2592-2605.

Grosh K, Zheng J, Zou Y, de Boer E, Nuttall AL (2004) High-frequency electromotile responses in the cochlea. J Acoust Soc Am 115:2178-2184.

Gummer AW, Hemmert W, Zenner HP (1996) Resonant tectorial membrane motion in the inner ear: its crucial role in frequency tuning. Proc Natl Acad Sci USA 93:8727-8732.

He DZZ, Dallos P (1999) Somatic stiffness of cochlear outer hair cells is voltage-dependent. Proc Natl Acad Sci USA 96:8223-8228.

He DZZ, Jia S, Dallos P (2004) Mechanoelectrical transduction of adult outer hair cells studied in a gerbil hemicochlea. Nature 429:766-770.
Hodgkin AL, Rushton WAH (1946) The electrical constants of a crustacean nerve fibre. Proc R Soc Lond B Biol Sci 133:444-479.

Hudspeth AJ, Corey DP (1977) Sensitivity, polarity, and conductance change in the response of vertebrate hair cells to controlled mechanical stimuli. Proc Natl Acad Sci USA 74:2407-2411.

Kössl M, Russell IJ (1992) The phase and magnitude of hair cell receptor potentials and frequency tuning in the guinea pig cochlea. J Neurosci 12:1575-1586.

Liberman MC, Gao J, He DZ, Wu X, Jia S, Zuo J (2002) Prestin is required for electromotility of the outer hair cell and for the cochlear amplifier. Nature 419:300-304.

Nuttall AL, Ren T (1995) Electromotile hearing: evidence from basilar membrane motion and otoacoustic emissions. Hear Res 92:170-177.

Nuttall AL, Dolan DF, Avinash G (1991) Laser Doppler velocimetry of basilar membrane vibration. Hear Res 51:203-213.

Preyer S, Renz S, Hemmert W, Zenner HP, Gummer AW (1996) Receptor potential of outer hair cells isolated from base to apex of the adult guineapig cochlea: implications for cochlear tuning mechanisms. Aud Neurosci 2:145-157.

Ren T (2002) Longitudinal pattern of basilar membrane vibration in the sensitive cochlea. Proc Natl Acad Sci USA 99:17101-17106.

Ruggero MA, Rich NC (1991) Furosemide alters organ of Corti mechanics: evidence for feedback of outer hair cells upon the basilar membrane. J Neurosci 11:1057-1067.

Santos-Sacchi J (1989) Asymmetry in voltage-dependent movements of isolated outer hair cells from the organ of Corti. J Neurosci 9:2954-2962.

Santos-Sacchi J (1992) On the frequency limit and phase of outer hair cell motility: effects of the membrane filter. J Neurosci 12:1906-1916.

Santos-Sacchi J, Dilger JP (1988) Whole cell currents and mechanical responses of isolated outer hair cells. Hear Res 35:143-150.

Sellick PM, Patuzzi R, Johnstone BM (1982) Measurement of basilar membrane motion in the guinea pig using the Mössbauer technique. J Acoust Soc Am 72:131-141.

Ulfendahl M, Khanna SM, Heneghan C (1995) Shearing motion in the hearing organ measured by confocal laser heterodyne interferometry. NeuroReport 6:1157-1160.

van Netten SM, Dinklo T, Marcotti W, Kros CJ (2003) Channel gating forces govern accuracy of mechano-electrical transduction in hair cells. Proc Natl Acad Sci USA 100:15510-15515.

von Békésy G (1951) Microphonics produced by touching the cochlear partition with a vibrating electrode. J Acoust Soc Am 23:29-35.

Zheng J, Shen W, He DZ, Long KB, Madison LD, Dallos P (2000) Prestin is the motor protein of cochlear outer hair cells. Nature 405:149-155.

Zwislocki JJ, Kletsky EJ (1979) Tectorial membrane: a possible effect on frequency analysis in the cochlea. Science 204:639-641. 